\title{
Observation of the out-of-plane magnetization in a mesoscopic ferromagnetic structure superjacent to a superconductor
}

Anna K. Suszka, Sebastian Gliga, Peter Warnicke, Sebastian Wintz, Susmita Saha, Kristin M. Charipar, Heungsoo Kim, Phillip Wohlhüter, Eugenie Kirk, Simone Finizio, Jörg Raabe, James D. S. Witt, Laura J.

Heyderman, and Nicholas S. Bingham

Citation: Appl. Phys. Lett. 113, 162601 (2018); doi: 10.1063/1.5051653

View online: https://doi.org/10.1063/1.5051653

View Table of Contents: http://aip.scitation.org/toc/apl/113/16

Published by the American Institute of Physics

\section{Articles you may be interested in}

Study of spin-orbit torque induced magnetization switching in synthetic antiferromagnet with ultrathin Ta spacer layer

Applied Physics Letters 113, 162402 (2018); 10.1063/1.5045850

Effect of $\mathrm{Cr}$ substitution on ferrimagnetic and ferroelectric properties of $\mathrm{GaFeO}_{3}$ epitaxial thin films Applied Physics Letters 113, 162901 (2018); 10.1063/1.5029442

Enhanced magnon spin transport in $\mathrm{NiFe}_{2} \mathrm{O}_{4}$ thin films on a lattice-matched substrate Applied Physics Letters 113, 162403 (2018); 10.1063/1.5049749

Direct-coupled micro-magnetometer with Y-Ba-Cu-O nano-slit SQUID fabricated with a focused helium ion beam Applied Physics Letters 113, 162602 (2018); 10.1063/1.5048776

Perpendicular magnetic anisotropy in bulk and thin-film CuMnAs for antiferromagnetic memory applications Applied Physics Letters 113, 162404 (2018); 10.1063/1.5048207

Stabilization and control of topological magnetic solitons via magnetic nanopatterning of exchange bias systems Applied Physics Letters 113, 162401 (2018); 10.1063/1.5047222

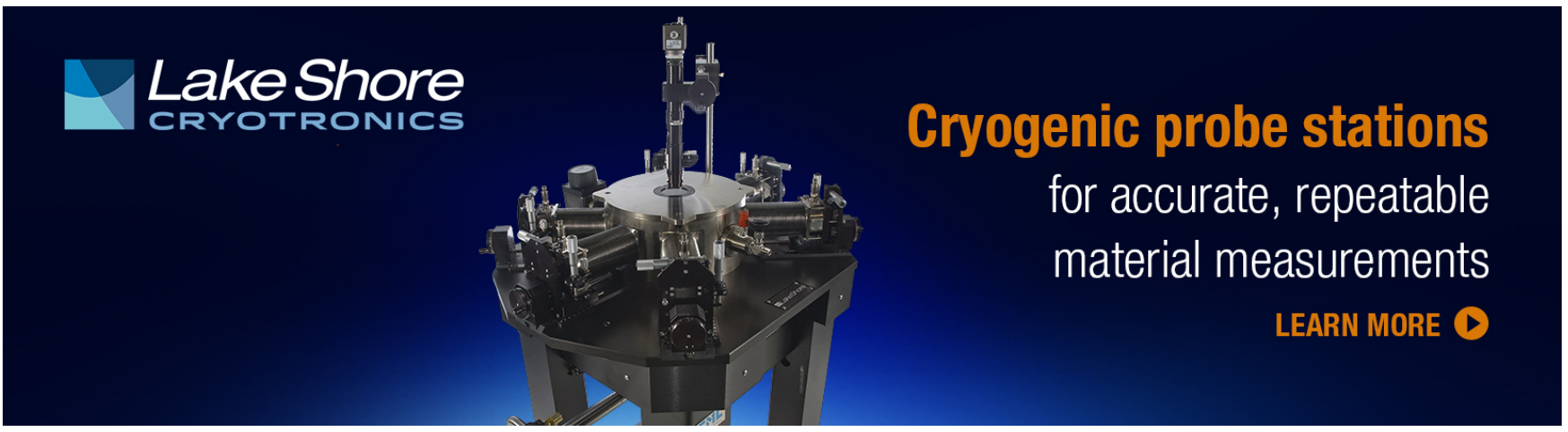




\title{
Observation of the out-of-plane magnetization in a mesoscopic ferromagnetic structure superjacent to a superconductor
}

\author{
Anna K. Suszka, ${ }^{1,2, a)}$ Sebastian Gliga, ${ }^{3}$ Peter Warnicke, ${ }^{2}$ Sebastian Wintz, ${ }^{2}$ \\ Susmita Saha, ${ }^{1,2}$ Kristin M. Charipar, ${ }^{4}$ Heungsoo Kim, ${ }^{4}$ Phillip Wohlhüter, ${ }^{1,2}$ \\ Eugenie Kirk, ${ }^{1,2}$ Simone Finizio, ${ }^{2}$ Jörg Raabe, ${ }^{2}$ James D. S. Witt, ${ }^{5}$ Laura J. Heyderman, ${ }^{1,2}$ \\ and Nicholas S. Bingham ${ }^{1,2,6, b)}$ \\ ${ }^{1}$ Laboratory for Mesoscopic Systems, Department of Materials, ETH Zürich, 8093 Zürich, Switzerland \\ ${ }^{2}$ Paul Scherrer Institut, 5232 Villigen PSI, Switzerland \\ ${ }^{3}$ SUPA, School of Physics and Astronomy, University of Glasgow, Glasgow G12 8QQ, United Kingdom \\ ${ }^{4}$ U.S. Naval Research Laboratory, Washington, DC 20375, USA \\ ${ }^{5}$ School of Physics and Astronomy, University of Leeds, Leeds LS2 9JT, United Kingdom \\ ${ }^{6}$ Department of Applied Physics, Yale University, New Haven, Connecticut 06511, USA
}

(Received 10 August 2018; accepted 26 September 2018; published online 15 October 2018)

\begin{abstract}
The geometry of magnetic flux penetration in a high temperature superconductor at a buried interface was imaged using element-specific x-ray excited luminescence. We performed low temperature observation of the flux penetration in $\mathrm{YBa}_{2} \mathrm{Cu}_{3} \mathrm{O}_{7-\delta}(\mathrm{YBCO})$ at a buried interface by imaging of the perpendicular magnetization component in square Permalloy (Py) mesostructures patterned superjacent to a YBCO film. Element specific imaging below the critical temperature of YBCO reveals a cross-like geometry of the perpendicular magnetization component which is decorated by regions of alternating out-of-plane magnetization at the edges of the patterned Py structures. The cross structure can be attributed to the geometry of flux penetration originating from the superconductor and is reproduced using micromagnetic simulations. Our experimental method opens up possibilities for the investigation of flux penetration in superconductors at the nanoscale. Published by AIP Publishing. https://doi.org/10.1063/1.5051653
\end{abstract}

While high temperature superconductors have been extensively studied for over 30 years, ${ }^{1}$ they continue to be a vivid research topic due to their numerous potential applications and complex physics. In this context, flux penetration in the presence of a magnetic field is a subject of intense investigation. Fundamental research in this area is driven by the study of vortex-vortex interactions, vortex propagation, or melting and pinning of a vortex lattice. Concurrently, industrial applications primarily use flux pinning as a means to generate high current densities. ${ }^{2}$

A multitude of techniques have been utilized for the real space observation of flux penetration in superconductors. ${ }^{3-7}$ While techniques such as scanning tunneling microscopy have high spatial resolution, ${ }^{8}$ they are limited in the scanning area. Conversely, imaging techniques based on the Faraday effect provide wavelength-limited resolution for large imaging areas. Recently, low temperature imaging of superconducting flux distribution with sub-50 nm spatial resolution over large areas $(>1 \mathrm{~mm})$ has been demonstrated using several element specific $\mathrm{x}$-ray techniques with surface sensitive detection techniques. ${ }^{9-12}$ However, such techniques are challenging due to difficulties in accessing the information from buried interfaces at temperatures below the superconducting critical temperature $\left(T_{C}\right)$. Measurements of the superconducting flux geometry often utilize a magnetic "control layer" deposited on top of a superconductor, where the respective magnetization state reflects the geometry of the flux. This indirect method is excellent for the detection of

\footnotetext{
a)suszka@gmail.com

b)binghamn@gmail.com
}

the micro- and nano-scale distribution of superconducting vortices where high resolution imaging is a prerequisite for their correct identification and interpretation. While a general understanding of flux penetration has been achieved, some of its microscopic details (e.g., vortex cutting and reconnection $^{13}$ and the role of defects in vortex creation ${ }^{14}$ ) still require further investigation. Additionally, while most reported experiments focus on the flux pattern, the detailed structure of the magnetization in the magnetic layer at the nanoscale remains unexplored. High precision imaging generates prospects of the discovery of interesting magnetization states in patterned nanostructures, resulting in exciting potential applications and emergent effects arising from the combination of magnetism and superconductivity. We present element specific measurements of the out-of-plane component of the magnetization in patterned $\mathrm{Py}\left(\mathrm{Ni}_{80} \mathrm{Fe}_{20}\right)$ mesostructures deposited on the high-temperature superconductor, $\mathrm{YBa}_{2} \mathrm{Cu}_{3} \mathrm{O}_{7-\delta}$ (YBCO), below $T_{C}$. We identify the main features of the observed magnetic state, which we attribute to the imprint of trapped magnetic flux in the subjacent superconductor.

For low temperature element-specific imaging of the magnetization state of the Py structures, we have used an $\mathrm{x}$ ray-excited optical luminescence (XEOL) technique and a scanning transmission $\mathrm{X}$-ray microscopy setup at the PolLux beamline, Swiss Light Source. ${ }^{15}$ In XEOL detection, the absorption of light by the sample is proportional to the intensity of the emitted luminescent signal from a substrate. This technique provides a thickness averaged signal and can be applied to investigate buried interfaces of films and multilayers. ${ }^{16-18}$ The schematic of the experimental setup is given 
in Fig. 1(a). The incident $\mathrm{x}$-ray beam is focused using a Fresnel zone plate (FZP) down to a $25 \mathrm{~nm}$ diameter spot. To guarantee that only the first order focus of the FZP illuminates the sample, an order selecting aperture (OSA), consisting of a $50 \mu \mathrm{m}$ diameter pinhole, is positioned in front of the FZP. The sample plane is perpendicular to the incident beam, and the luminescent signal is recorded using a photomultiplier tube (PMT, Hamamatsu R1463P). The sample environment and the cooling setup [Fig. 1(b)] consist of a liquid $\mathrm{N}_{2}$ constant flow cryostat. The temperature of the sample is monitored using two thermometers: one located close to the copper cooling element $\left(T_{1}\right)$ and the other below the sample $\left(T_{2}\right)$. During the imaging, we continuously record the resistance of the YBCO so as to ensure that the sample remains in the superconducting state throughout the measurements.

The sample is schematically presented in Fig. 2(a) and consists of $\mathrm{MgO}$ (100) (substrate)/YBCO $(150 \mathrm{~nm}) / \mathrm{Al}$ $(1.0 \mathrm{~nm}) / \mathrm{Py}(25 \mathrm{~nm}) / \mathrm{AlN}(100 \mathrm{~nm}) / \mathrm{Cu}_{\text {stripline }}(80 \mathrm{~nm})$. YBCO was grown using pulsed laser deposition via a pulsed excimer laser $(\lambda=248 \mathrm{~nm}$ and pulse width $=20 \mathrm{~ns})$ at a fixed target to a substrate distance of $4 \mathrm{~cm}$ and a laser fluence of $F=2 \mathrm{~J} / \mathrm{cm}^{2}$ at a constant substrate temperature of $990 \mathrm{~K}$ in an $\mathrm{O}_{2}$ partial pressure of $p_{\mathrm{O}_{2}}=400 \mathrm{mTorr}$. After the deposition, the sample was annealed at $990 \mathrm{~K}$ in $p_{O_{2}}=300$ Torr for 90 min. The Al/Py/AlN stack was deposited on top of YBCO using evaporation (Al, Py) and sputtering (AlN). Thicknesses were calibrated using atomic force microscopy (AFM) and scanning electron microscopy (SEM). Py squares with side lengths varying from $2 \mu \mathrm{m}$ to $6 \mu \mathrm{m}$ were fabricated on the same sample using electron-beam lithography followed by liftoff. Prior to x-ray imaging, the sample morphology was characterized using AFM and SEM. The electrical properties of the sample were measured using a Quantum Design physical property measurement system (PPMS), while magnetic properties were measured using a magneto-optical Kerr effect (nanoMOKE3) setup and a Quantum Design superconducting quantum interference device-vibrating sample magnetometer (SQUID-VSM, MPMS3).

The room temperature nanoMOKE hysteresis loop [Fig. 2(b)] of the squares indicates the absence of the expected Landau domain pattern ${ }^{19}$ for which remanent magnetization of the flux closure state is zero at zero applied field. ${ }^{20}$ Instead, the hysteresis loop exhibits nearly full remanent magnetization after saturation with a field of the order of $\mu_{0} \mathrm{H} \approx 15 \mathrm{mT}$ and a more complex domain-mediated magnetization reversal

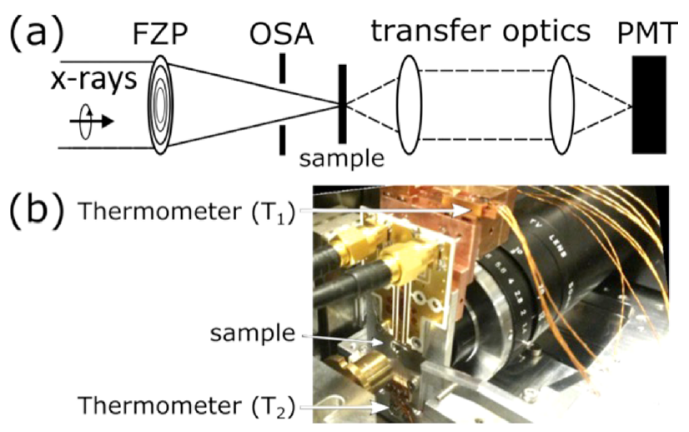

FIG. 1. (a) Schematic drawing of the optics used for low temperature XEOL imaging at the PolLux beamline and (b) image of the sample location and experimental setup. The positions of thermometers and sample are indicated.

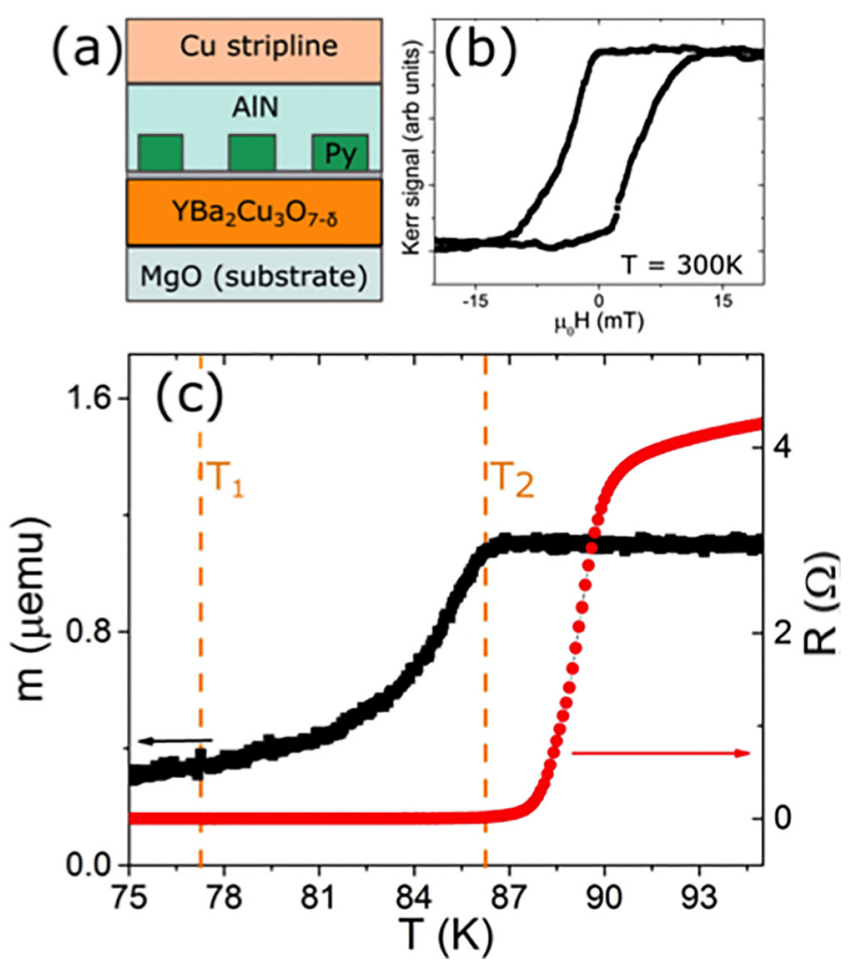

FIG. 2. (a) Schematic of the sample. (b) Room temperature in-plane MOKE hysteresis loop of a $6 \mu \mathrm{m}$ square. (c) Resistance (red dots) and volumeaveraged magnetic moment (black squares) of the measured sample as a function of temperature. Arrows indicate the respective axes. Orange dashed lines indicate the temperatures recorded using two thermometers: $\mathrm{T}_{1}$ and $\mathrm{T}_{2}$ for XEOL measurements.

process. ${ }^{21}$ The lack of a Landau pattern is most likely due to significant roughness of the underlying YBCO film $\left(\delta_{\mathrm{RMS}}\right.$ $\approx 21 \mathrm{~nm}$ ), which prevents the magnetization in Py from relaxing into the expected, lowest energy state. The $T_{\mathrm{C}}$ of the YBCO thin film was measured via four point probe resistance and volume-averaged magnetization measurements as a function of temperature [Fig. 2(c)]. The drop in resistance and onset of diamagnetism indicate $T_{C} \approx(87.0 \pm 0.3) \mathrm{K}$. The $T_{C}$ of the film differs from bulk YBCO $\left[T_{C_{\text {bulk }}}=90.2 \mathrm{~K}\right.$ (Ref. 22)] which is most likely due to large lattice mismatches between the $\mathrm{MgO}$ substrate and the YBCO film, giving rise to slight stoichiometric and crystalline defects, finite size effects, and residual strain. A large lattice mismatch between the film and substrate also leads to high surface roughness which facilitates the multi-domain magnetization state of the Py structures.

In Figs. 3(a) and 3(b), room temperature SEM and unpolarized XEOL images below $T_{C}$ of a representative array of

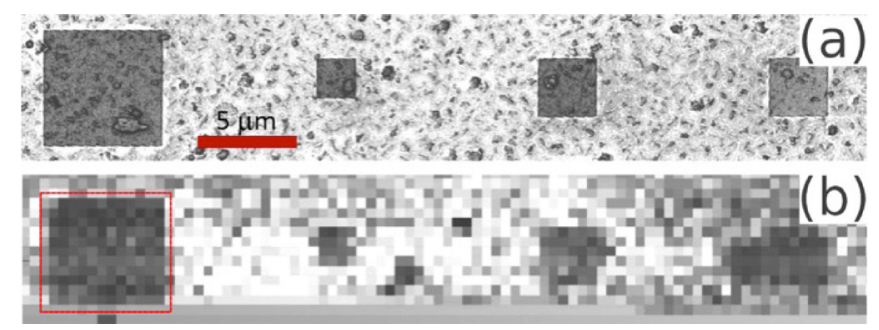

FIG. 3. (a) SEM and (b) XEOL images of a representative array of patterned Py squares on top of the YBCO thin film. 
Py squares, respectively, are shown. The experimental results shown in Figs. 4(a) and 4(c) are taken from the $6 \mu \mathrm{m}$ square indicated with the red frame in Fig. 3(b). Here, we identify the low temperature magnetization state using the $\mathrm{x}$ ray magnetic circular dichroism (XMCD) effect. ${ }^{23}$ This method yields a signal intensity which is proportional to the spin dependent density of states at the Fermi level and consequently to the effective spin polarization of the studied material at a particular $\mathrm{x}$-ray absorption edge. The XMCD ratio $\left(\mathrm{I}_{\mathrm{XMCD}}\right)$ is calculated using the difference between the normalized intensities of right $\left(I_{c^{+}}\right)$and left $\left(I_{c^{-}}\right)$handed circularly polarized light $\left[\mathrm{I}_{\mathrm{XMCD}}=\left(\mathrm{I}_{\mathrm{c}^{+}}-\mathrm{I}_{\mathrm{c}^{-}}\right) /\left(\mathrm{I}_{\mathrm{c}^{+}}+\mathrm{I}_{\mathrm{c}^{-}}\right)\right]$. Our experimental setup provides sensitivity to the out-of-plane magnetization component with the measurements taken at the $\mathrm{Ni}_{3}$ edge $(852.7 \mathrm{eV})$. All of the images were recorded below $T_{C}$ of the YBCO film. Before imaging, the sample was briefly subjected to an out-of-plane magnetic field from the $\mathrm{Cu}$ stripline, in order to induce the vortex state in the YBCO, while subsequent measurements were taken at an external magnetic field of a few $\mathrm{mT}$. Visible in the XMCD image [Fig. 4(a)] is a dark "cross" pattern formed by narrow regions with pronounced out-of-plane magnetization within the brighter Py square. We note that the outline of the cross roughly overlaps with the diagonals of the square although, due to pinning, it is not perfectly centered, as illustrated in the schematic drawing in Fig. 4(b). Each diagonal is terminated by regions of alternating out-of-plane contrast, leading to local dark and bright spots. The bottom edge of the square is "decorated" by an irregular pattern of white spots with an average diameter of $d_{d o t} \approx(550 \pm 70) \mathrm{nm}$. In order to demonstrate that the contrast seen in the XMCD signal is not due to film morphology, we show the addition $\left(\mathrm{I}_{\mathrm{c}^{+}}+\mathrm{I}_{\mathrm{c}^{-}}\right)$in Fig. 4(c), which exhibits no "cross" pattern or alternating bright and dark regions outside of the Py structure.

Typically, the magnetization in mesoscopic Py square structures forms a flux-closure or Landau state ${ }^{19}$ characterized by four domains with orthogonal magnetization. These
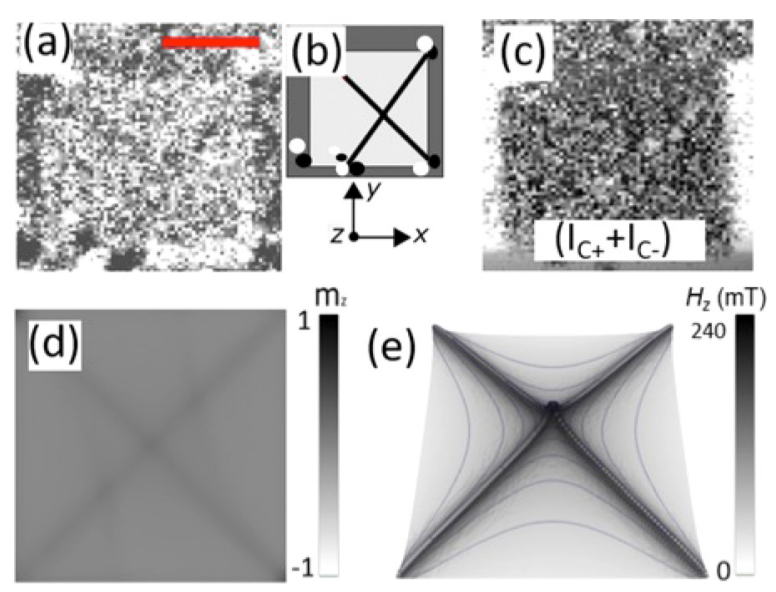

FIG. 4. (a) XMCD image of the Py square recorded at the $\mathrm{Ni} \mathrm{L}_{3}$ edge and (b) a schematic representation showing a dark cross on top of the Py square corresponding to the out-of-plane magnetization component, as well as the alternating out-of-plane magnetization pattern along the perimeter of the square. (c) The summation of circular right and left polarization images of the Py square showing no magnetic contrast. (d) Simulated out-of-plane magnetization in the Py square in the presence of the field originating from the superconductor. (e) Simulated field distribution generated by the supercurrent circulating in the YBCO. domains are separated by Néel walls that form a similar cross pattern. However, the existence of a Landau state is unlikely since the magnetization measurements [Fig. 2(b)] indicate a multi-domain magnetization state of the Py elements at room temperature.

Therefore, the observation of a cross-shaped magnetization component below the $T_{C}$ of a ferromagnet superjacent to a superconductor can be reasonably attributed to the distribution of the magnetic flux in the superconductor. ${ }^{24-26}$ It has been shown that the geometry of the trapped flux and the distribution of a supercurrent depend on the geometry of a superconducting sample. For a square-shaped sample, the current runs parallel to the sides of the square and exhibits sharp folds along the diagonals called "discontinuity lines." This is associated with the spatial distribution of electric and magnetic fields which is imprinted onto the magnetization profile of the superjacent magnetically soft mesostructure ${ }^{24}$ [Fig. 4(a)]. The terminating out-of-plane magnetized regions [white and dark spots around the edges of the square in Fig. 4(a)] most likely correspond to localized flux patches and have, thus far, not been experimentally observed nor mathematically described within available flux penetration theory. This is because in most experimental and theoretical cases the boundaries of superconducting and ferromagnetic films coincide, ${ }^{12}$ therefore offering no way of quantifying edge effects in finite sized magnets.

To understand the effect of the highly inhomogeneous magnetic field of the superconductor on the Py sample, we have performed fully three-dimensional micromagnetic simulations based on a hybrid finite-element/boundary-element method. ${ }^{27}$ The Py structure was discretized using a tetrahedral mesh with an average edge length of $5 \mathrm{~nm}$. The material parameters for Permalloy were saturation polarization $\mu_{0} M_{S}$ $=1 \mathrm{~T}$, exchange constant $A=1.3 \times 10^{-11} \mathrm{~J} \mathrm{~m}^{-1}$, and no crystalline anisotropy, $K=0 \mathrm{~J} \mathrm{~m}^{-3}$. To reduce computing time, the simulated sample was reduced in dimensions, with a side length of $500 \mathrm{~nm}$, while keeping the $25 \mathrm{~nm}$ thickness as in the experiment. The out-of-plane field [represented in Fig. 4(e)], $H_{z}$, generated by the supercurrent circulating in the superconductor is described by ${ }^{24}$

$$
\begin{gathered}
H_{z}(x, y)=H_{a}+\frac{J_{c}}{4 \pi} \sum_{p, q= \pm 1} f(p x, q y), \\
f(x, y)=\sqrt{2} \ln \frac{\sqrt{2} P+a+b-x-y}{\sqrt{2} Q-a+b-x-y} \\
+\ln \left|\frac{(P+y-b)(y-b+a)(P+x-a) x}{(y-b)(Q+y-b+a)(x-a)(Q+x)}\right|,
\end{gathered}
$$

where $P=\left[\left(a-x^{2}+(b-y)^{2}\right)\right]^{1 / 2}, Q=\left[x^{2}+(b-a-y)^{2}\right]^{1 / 2}$, $J_{c}=4 \times 10^{11} \mathrm{~A} / \mathrm{m}^{2}$ is the critical current density, and $a$ and $b$ are the side lengths of the sample. $H_{a}$ is a small (ca. $10 \mathrm{mT}$ ) constant offset field.

The effect of the field in Eq. (1) on the out-of-plane component of the magnetic state, $m_{z}$, is shown in Fig. 4(d), where $m_{z}=M_{z} / M_{s}$. The simulations reproduce the experimentally observed cross shape, where the cross is symmetric about the center of the Py square in the absence of pinning. Interestingly, the simulations also show that the cross 
structure is present irrespective of the initial in-plane magnetic configuration and does not lead to the formation of a Landau magnetic state in the Py square. In fact, we find that the observed pattern only locally modifies the magnetic structure, around the region of the cross, leaving the initial pattern mostly unchanged. While the simulations do not reproduce the experimentally observed peripheral regions with alternating out-of-plane magnetization, we believe that these are due to a combination of the localization of supercurrent in the region below the Py square (given that the magnetic square is patterned on top of an extended superconductor) and pinning sites caused by the overall roughness of the film. Therefore, we expect enhanced pinning of the supercurrent, in particular at the edges of the patterned square. The alternating contrast (and hence the $m_{z}$ component) at the edges is consistent with flux closure.

In conclusion, using scanning transmission $\mathrm{x}$-ray microscopy, we have imaged the low temperature out-ofplane magnetization component of a mesoscopic Py square superjacent to a superconducting YBCO thin film at a buried interface. The observed cross-shaped magnetization pattern is attributed to the geometry of the magnetic flux penetration in YBCO and is qualitatively confirmed with micromagnetic simulations. We also observed small out-of-plane magnetized regions surrounding the Py square, which are attributed to the complex magnetic stray field surrounding the Py square. The physics behind the observed magnetization states at superconducting-ferromagnetic interfaces has potentially significant implications for the understanding of the mixed state in superconductors and for the manipulation of the spin structure in coupled ferromagnetic and superconductor heterostructures.

We would like to thank Ch. Jooss and G. Burnell for helpful discussion of the results, C. Vaz, M. Radovic, and M. Horisberger for help with sample preparation, A. Weber, V. Guzenko, and E. Deckardt for help with the sample processing, and B. Sarafimov for technical help during the experiment. A.K.S. acknowledges funding from the European Community's Seventh Framework Program (FP7/ 2007-2013) under Grant Agreement No. 290605 (COFUND: PSI-FELLOW). Phillip Wohlhüter was supported by the Swiss National Science Foundation. S.G. was funded by the European Union's Horizon 2020 research and innovation programme under Marie Skłodowska-Curie Grant Agreement No. 708674. S.S. acknowledges the ETH Zurich Post Doctoral fellowship and Marie Curie actions for the People COFUND program (Grant No. FEL-11 16-1). Part of this work was performed at the PolLux (X07DA) endstation of the Swiss Light Source, Paul Scherrer Institut, Villigen, Switzerland. S.G. thanks A. Kákay for helpful discussions.

${ }^{1}$ J. G. Bednorz and K. A. Müller, Z. Phys. B: Condens. Matter 64, 189 (1986).

${ }^{2}$ M. Tomita and M. Murakami, Nature 421, 517 (2003).

${ }^{3}$ H. Darhmaoui, J. Jung, J. Talvacchio, M. A.-K. Mohamed, and L. Friedrich, Phys. Rev. B 53, 12330 (1996).

${ }^{4}$ L. Thiel, D. Rohner, M. Ganzhorn, P. Appel, E. Neu, B. Müller, R. Kleiner, D. Koelle, and P. Maletinsky, Nat. Nanotechnol. 11, 677 (2016).

${ }^{5}$ C. A. Durän, P. L. Gammel, R. Wolfe, V. J. Fratello, D. J. Bishop, J. P. Rice, and D. M. Ginsberg, Nature 357, 474 (1992).

${ }^{6}$ S. J. Bending, P. J. Curran, W. M. A. Desoky, V. V. Khotkevych, A. Gibbs, A. P. Mackenzie, T. Tamegai, and S. E. Sebastian, Physica C 479, 65 (2012).

${ }^{7}$ J. R. Kirtley, Rep. Prog. Phys. 73, 126501 (2010).

${ }^{8}$ T. Nishio, T. An, A. Nomura, K. Miyachi, T. Eguchi, H. Sakata, S. Lin, N. Hayashi, N. Nakai, M. Machida, and Y. Hasegawa, Phys. Rev. Lett. 101, 167001 (2008).

${ }^{9}$ S. Ruoss, C. Stahl, M. Weigand, G. Schutz, and J. Albrecht, Appl. Phys. Lett. 106, 022601 (2015).

${ }^{10}$ C. Stahl, S. Ruoss, M. Weigand, M. Bechtel, G. Schutz, and J. Albrecht, J. Appl. Phys. 117, 17D109 (2015).

${ }^{11}$ C. Stahl, P. Audehm, J. Gräfe, S. Ruoss, M. Weigand, M. Schmidt, S. Treiber, M. Bechtel, E. Goering, G. Schütz, and J. Albrecht, Phys. Rev. B 90, 104515 (2014).

${ }^{12}$ A. Palau, S. Valencia, N. Del-Valle, C. Navau, M. Cialone, A. Arora, F. Kronast, D. A. Tennant, X. Obradors, A. Sanchez, and T. Puig, Adv. Sci. 3, 1600207 (2016).

${ }^{13}$ V. Vlasko-Vlasov, A. Koshelev, A. Glatz, C. Phillips, U. Welp, and W. Kwok, Phys. Rev. B 91, 014516 (2015).

${ }^{14}$ S. Ruoss, C. Stahl, M. Weigand, P. Zahn, J. Bayer, G. Schuetz, and J. Albrecht, New J. Phys. 18, 103044 (2016).

${ }^{15}$ J. Raabe, G. Tzvetkov, U. Flechsig, M. Boge, A. Jaggi, B. Sarafimov, M. G. C. Vernooij, T. Huthwelker, H. Ade, D. Kilcoyne, T. Tyliszczak, R. H. Fink, and C. Quitmann, Rev. Sci. Instrum. 79, 113704 (2008).

${ }^{16}$ C. A. F. Vaz, C. Moutafis, M. Buzzi, and J. Raabe, J. Electron Spectrosc. Relat. Phenom. 189, 1 (2013).

${ }^{17}$ C. A. F. Vaz, C. Moutafis, C. Quitmann, and J. Raabe, Appl. Phys. Lett. 101, 083114 (2012).

${ }^{18}$ R. A. Rosenberg, S. Zohar, D. Keavney, R. Divan, D. Rosenmann, A. Mascarenhas, and M. A. Steiner, Rev. Sci. Instrum. 83, 073701 (2012).

${ }^{19}$ J. Raabe, C. Quitmann, C. H. Back, F. Nolting, S. Johnson, and C. Buehler, Phys. Rev. Lett. 94, 217204 (2005).

${ }^{20}$ R. Antos, Y. Otani, and J. Shibata, J. Phys. Soc. Jpn. 77, 031004 (2008).

${ }^{21}$ K. D. Sattler, Handbook Nanophysics: Nanoelectronics Nanophotonics (Taylor and Francis, 2011), Vol. 104, p. 083909.

${ }^{22}$ R. J. Cava, B. Batlogg, R. B. van Dover, D. W. Murphy, S. Sunshine, T. Siegrist, J. P. Remeika, E. A. Rietmann, S. Zahurak, and G. P. Espinosa, Phys. Rev. Lett. 58, 1676 (1987).

${ }^{23}$ G. Schütz, W. Wagner, W. Wilhelm, and P. Kienle, Phys. Rev. Lett. 58, 737 (1987).

${ }^{24}$ E. H. Brandt, Phys. Rev. B 52, 15442 (1995).

${ }^{25}$ Ch. Jooss, J. Albrecht, H. Kuhn, S. Leonhardt, and H. Kronmüller, Rep. Prog. Phys. 65, 651 (2002).

${ }^{26}$ E. H. Brandt, Phys. Rev. Lett. 74, 3025 (1995).

${ }^{27}$ A. Kákay, E. Westphal, and R. Hertel, IEEE Trans. Magn. 46, 2303 (2010). 